Research Article

\title{
Simulating Study on Mechanical Properties of Rock Wool Board for Thermal Insulation on External Walls
}

\author{
Xiaoguang Li (iD, Jinjin Liu, Xin Fan, Jinyi Qin, Rui Zhang, Ren Cao, Panqi Wang, \\ and Xiaoliang Huo \\ School of Civil Engineering, Chang'an University, Xi'an 710061, China \\ Correspondence should be addressed to Xiaoguang Li; gxleee@chd.edu.cn
}

Received 15 August 2019; Revised 9 October 2019; Accepted 26 November 2019; Published 12 February 2020

Academic Editor: Victor M. Castaño

Copyright ( $) 2020$ Xiaoguang Li et al. This is an open access article distributed under the Creative Commons Attribution License, which permits unrestricted use, distribution, and reproduction in any medium, provided the original work is properly cited.

\begin{abstract}
Rock wool board (RWB) is widely used in construction of exterior insulation worldwide. Fiber diameter, solid volume fraction (SVF), and contact degree among the fibers significantly influence the RWB physical properties. Herein, the effects of these factors on the mechanical properties of RWB were investigated using the GeoDict software. First, the fiberization process resulted in a finer fiber diameter, and the SVF of RWB increased with decreasing pore sizes. In addition, both the fiber diameter and SVF significantly influenced the RWB shear strength. Furthermore, in compliance with the Chinese standards of compression, tensile, and shear strength, the SVF of RWB with a $10.5 \mu \mathrm{m}$ fiber diameter did not exceed $4.72 \%$, $4.04 \%$, and $5.4 \%$, respectively. The novel method proposed herein can be used for optimizing the RWB manufacturing process.
\end{abstract}

\section{Introduction}

As an insulating material, rock wool board (RWB) is widely used for exterior insulation. Over the past few decades, the thermal conductivity and mechanical and physical performance requirements of this material have been significantly improved. However, detailed research of the mechanical properties of fiber products with complex mesostructures faces great challenges, as the traditional macroscopic test cannot accurately predict deformation behavior of fiber products or recommend optimized mesoscopic structural parameters (such as fiber density, length, diameter, and contact point) [1].

RWB is composed of different-sized fibers that are connected by simple overlap. The connection between fibers and the influence of resin on the strength and rigidity of RWB are significant [2]. Bond failure between fibers and frictional sliding also greatly influence deformation and damage of RWB, which have been experimentally observed by Liu et al. and Wilbrink et al. $[3,4]$. RWB deteriorates over time, and the bond point between the RWB and exterior plastering layer was invalid, causing the covering layer to peel off. Because of the large negative wind pressure $[5,6]$, insulation of the external building walls (Figure 1) can fall off or even damage external insulation systems. Therefore, different requirements are imposed on the mechanical properties of RWB depending on its desired use.

For practical applications, RWB requires different strengths to resist environment forces and its own action. In the field of composite insulation boards for external walls, the shear and tensile stresses of intermediate layers of RWB were relatively large due to external environment, which significantly influenced the strength characteristics of RWB under mutually perpendicular shear loads [7]. The compressive strength and other mechanical properties of mineral wool products depended on the distribution of fibers in the structure, as well as the direction of load action and product density [8]. When the fiber product is subjected to a load and the local strain is not uniform, local damage can occur [9]. However, few studies have been reported on its mechanical properties. Some studies have used numerical simulation to examine the relationship between the RWB mesostructure and macroscopic performance. The research and design of composite mesostructure plays a key role in material design [10-12].

To study the correlation between the mesostructure and mechanical properties of RWB, the mechanical properties of 


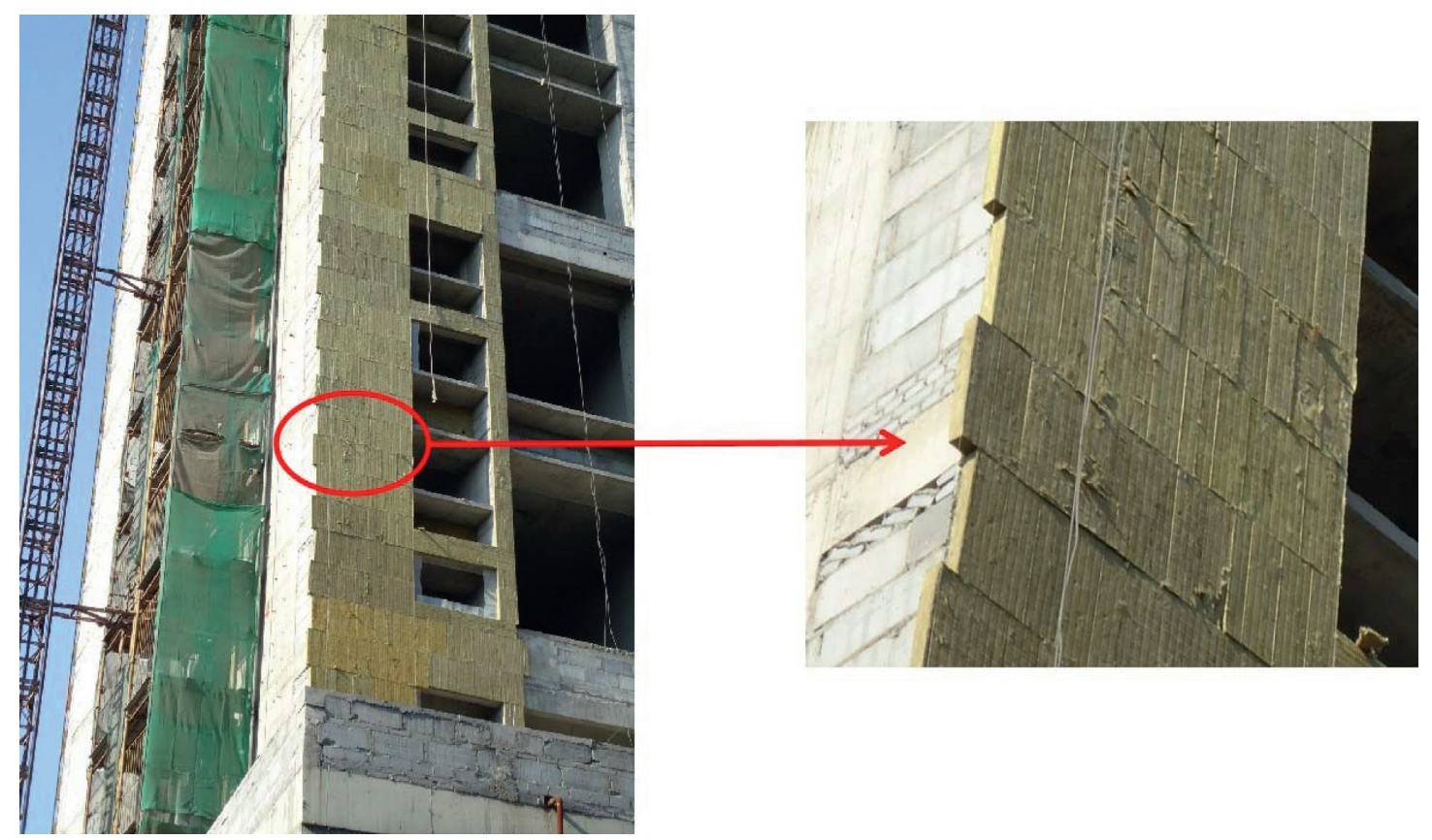

FIgURE 1: The installation process of RWB.

different mesostructured RWBs can be calculated by numerical simulation [13]. X-ray tomography (CT) [14-16] has been used to obtain scanned images of fiber products, which are subsequently imported into GeoDict software to determine the real structure of fiber products, calculate macroscopic deformation ability [17, 18], and predict the mechanical properties [19] of the fiber products. Equipped with an improved algorithm $[20,21]$ to establish a $3 \mathrm{D}$ fiber structure model of continuous long fibers and short fibers, the relationship between fiber length, diameter, density, and orientation was studied.

The compression, tensile, and shear strength of RWB were also tested using a WDW3030 microcontrol electronic universal testing machine (UTM; Kexin Testing Instrument Co. Ltd., WDW3030, Changchun, China). Combined with the software approach, the compressive, tensile, and shear strengths of RWB with different fiber diameters, solid volume ratios, and degrees of contact were calculated. Fiber diameter was $3-10.5 \mu \mathrm{m}$, and the solid volume ratio was $3.70-6.08 \%$. The formula for optimizing the strength index of RWB was also determined. This research lays the foundation for optimization of RWB structural design and optimization of industrial manufacturing.

\section{Materials and Methods}

2.1. Materials. The RWB was an inorganic fiber glass product [22] based on natural rocks (such as basalt) as the main raw material, containing a certain amount of admixtures. A series of processes, including melting at high temperature [23, 24] (Figure 2(a)), four-roll high-speed centrifuge spinning $[25,26]$ (Figure 2(b)), fiberization processing [23], postprocessing, and other processes were performed, and the chemical composition is listed in Table 1.
2.1.1. Elemental Analysis. The main constituent elements of the fiber were $\mathrm{Si}, \mathrm{Al}, \mathrm{Ca}$, and $\mathrm{Mg}$, which account for approximately $82.08 \%$ of the total content. In addition, a small amount of $\mathrm{Na}, \mathrm{P}, \mathrm{K}, \mathrm{Ti}, \mathrm{Mn}$, and Fe were detected. Since $\mathrm{Si}^{4+}$ and $\mathrm{Al}^{3+}$ were the main components of the network forming the fibers, which together constituted the skeleton, high content of oxides such as $\mathrm{SiO}_{2}$ and $\mathrm{Al}_{2} \mathrm{O}_{3}$ contributed to improved fiber stability [22]. In addition, oxides such as $\mathrm{MgO}$ and $\mathrm{CaO}$ acted as network-modified ions, and the filled fiber structure and network forming ions constitute the vitreous structure.

\subsection{Computational Methods}

\subsubsection{Experiment}

(1) Elastic Modulus. A YG005E electronic single fiber strength machine (Fangyuan Instrument Co., Ltd., YG005E, Wenzhou, China) was used to measure the tensile strength of the single fibers. The single fiber strength machine had a range of $50 \mathrm{cN}$ and a graduation value of $0.01 \mathrm{cN}$. The upper and lower jaws of the machine were set at a distance of $50 \mathrm{~mm}$, and the tensile speed was $5.0 \mathrm{~mm} / \mathrm{min}$. The mean tensile strength of the fibers was measured, as shown in Table 2, and the elastic modulus of a single fiber was 61.4 GPa:

$$
\sigma=\frac{F \times 10^{-4}}{\pi(D / 2)^{2}}
$$

where $\sigma$ is to the tensile strength of the monofilament, $\mathrm{MPa}$; $F$ is the monofilament breakage force, $\mathrm{cN}$; and $D$ is the mean diameter, $\mu \mathrm{m}$. 


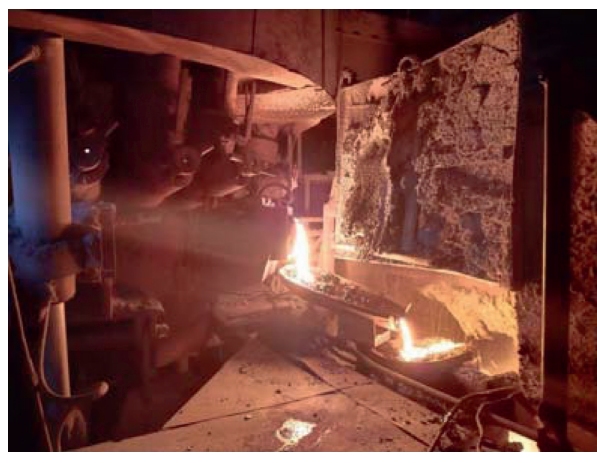

(a)

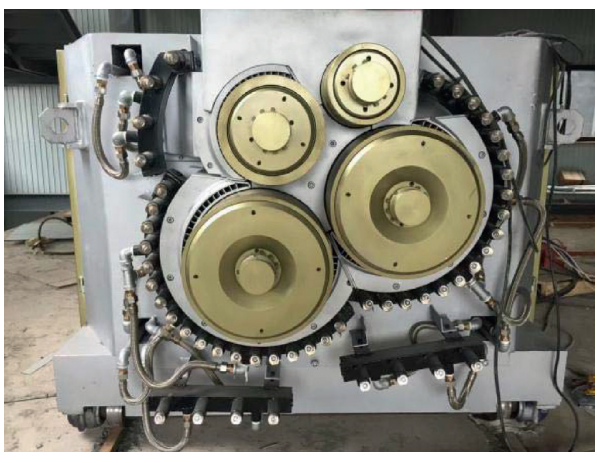

(b)

Figure 2: (a) High-temperature furnace and (b) four-roll high-speed centrifuge.

TABle 1: Chemical composition of the examined fibers.

\begin{tabular}{lcccccc}
\hline Composition & $\mathrm{SiO}_{2}$ & $\mathrm{Al}_{2} \mathrm{O}_{3}$ & $\mathrm{MgO}$ & $\mathrm{CaO}$ & $\mathrm{TFe}_{2} \mathrm{O}_{3}$ & $\mathrm{~K}_{2} \mathrm{O}$ \\
\hline Content (\%) & 37.37 & 13.08 & 10.13 & 21.50 & 6.63 & 1.42 \\
Composition & $\mathrm{Na}_{2} \mathrm{O}$ & $\mathrm{TiO}_{2}$ & $\mathrm{P}_{2} \mathrm{O}_{5}$ & $\mathrm{MnO}$ & LOI & TOTAL \\
Content (\%) & 2.96 & 2.42 & 0.32 & 0.20 & 2.96 & 98.72 \\
\hline
\end{tabular}

(2) Mechanical Properties. Considering China's requirements for strength, the RWB specimens were prepared. The specimens had dimensions of $100 \mathrm{~mm} \times 100 \mathrm{~mm} \times 30 \mathrm{~mm}$ and $200 \mathrm{~mm} \times 100 \mathrm{~mm} \times 30 \mathrm{~mm}$, and the SVF values were $3.70 \%$, $4.04 \%, 4.38 \%, 4.72 \%, 5.06 \%, 5.4 \%, 5.74 \%$, and $6.08 \%$, respectively. The samples were dried to constant weight in a 101-1-type hot constant temperature blast drying at approximately $105^{\circ} \mathrm{C}$ and then removed and placed in an environment of $(23 \pm 5)^{\circ} \mathrm{C}$ for $6 \mathrm{~h}$. Afterwards, each strength value reported was the average of three samples. Strength was examined using a WDW3030 microcontrol electronic UTM (Kexin Testing Instrument Co., Ltd., Changchun, China).

To measure the compressive strength, the RWB was mounted on a press and a prepressure of $250 \mathrm{~Pa}$ was applied with a constant speed of $0.1 \mathrm{~d} / \mathrm{min}( \pm 25 \%$ or less) until the sample yielded or compressed to $10 \%$ deformation to obtain compressive strength.

The tensile strength was measured with the sample pasted on two rigid plates with marble glue and curing agent. The sample was subsequently mounted on the test machine fixture and loaded at a constant speed of $(10 \pm 1) \mathrm{mm} / \mathrm{min}$ until it was destroyed to obtain its tensile strength.

To measure the shear strength, the sample was bonded to the fixture with marble glue and curing agent, and the fixture was fixed on the UTM and loaded with a speed of $(3 \pm 0.5)$ $\mathrm{mm} / \mathrm{min}$ along the length parallel to the sample. The rigid support plate transmitted shear stress to the sample, allowing the sample to be sheared until it was broken to obtain the shear strength.

Because of the complexity of the fiber products, it was not possible to quantitatively analyze the influence of fiber diameter on mechanical properties in laboratory tests. Therefore, two RWBs (Figure 3) with different diameter distributions with SVF of $4.72 \%$ were selected for qualitative analysis to study the influence of fiber diameter on the mechanical properties of RWB.

\subsubsection{Numerical Simulation}

(1) CT Scanning. The studied RWB was a cube with a side length of $2 \mathrm{~mm}$. The sample was scanned using a nanotom $\mathrm{m}$ CT (phoenix nanotom $m$ CT, Zeiss, Germany) with a $180 \mathrm{kV} / 15 \mathrm{~W}$ high-power nanofocus X-ray tube and detail detectability to $200 \mathrm{~nm}$. CT images were photographed, and the SVF was $4.72 \%$. The regular fiber distribution was similar in three directions (part 2 in the Supplementary Material).

(2) Simulation Method. The diameter of RWB was extracted by the FiberGuess module and was in accordance with a Gaussian distribution, with a mean diameter of $10.5 \mu \mathrm{m}$. The original model was established by Import Module in the software. To simplify calculation, the FiberGeo module was used based on the original model to input the main parameters (SVF, fiber length, diameter, cross-sectional shape, and fiber overlapping method) that can directly represent the geometric characteristics of the material to establish a simplified RWB model. Finally, the ElastoDict module was used to calculate the mechanical properties of RWB with different mesostructures (Figure 4).

2.3. Theory. GeoDict software was used to analyze the mechanical properties of RWB due to its complex force characteristics. An appropriate size of representative volume element (RVE) [27, 28] was selected to represent the actual behavior of the mesostructure, constructed using experimental data of fiber length, diameter, and orientation. After the mechanical model was established, the basic solution equation was obtained. The equivalent elastic modulus was obtained by using the Green periodic condition and mathematical transformation.

Application of the L-S equation based on the FFT method can accurately calculate the local stress and strain in a fiber network. Therefore, the numerical simulations used the L-S equation based on periodic Green's function of FFT to calculate the mechanical index of the RWB model as follows:

$$
\varepsilon(x)=E-\left(\Gamma^{0} \times \tau\right)(x), \quad x \in \Omega,
$$

where $\varepsilon(x)$ refers to the strain of the model on $\Omega$, in which $\Omega$ is the unit body that is the boundary condition; $E$ is the 
TABLE 2: Mean tensile strength of the investigated fibers.

\begin{tabular}{ccccc}
\hline & Diameter $(\mu \mathrm{m})$ & Breaking force $(\mathrm{cN})$ & Strength $(\mathrm{MPa})$ & Standard deviation $(\%)$ \\
\hline Fiber & 9.867 & 8.17 & 1068.50 & 3.7 \\
\hline
\end{tabular}

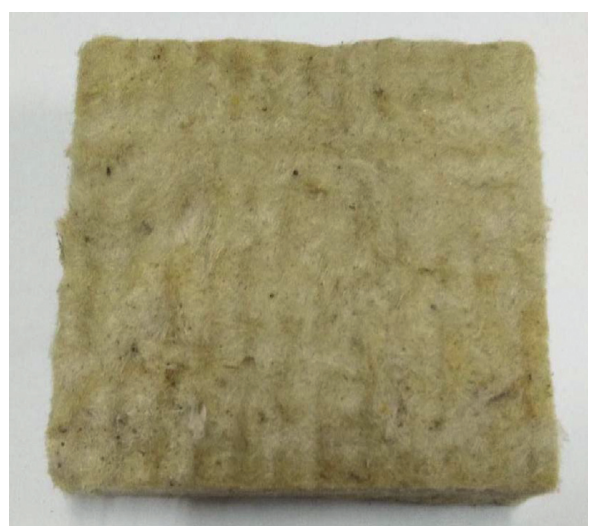

(a)

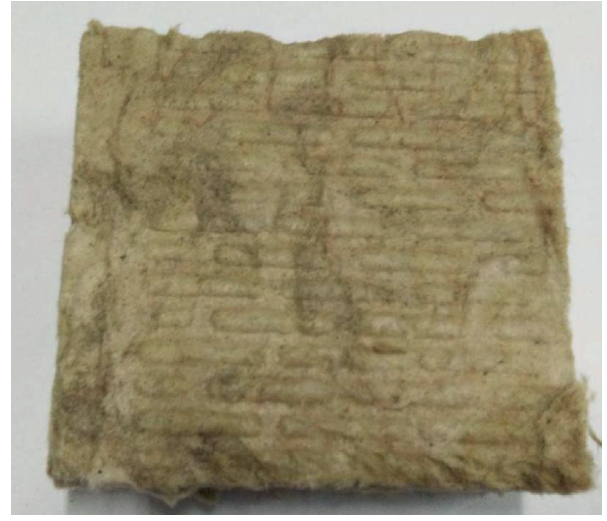

(b)

Figure 3: RWB samples with different diameter distributions: (a) mean diameter $=10.5 \mu \mathrm{m}$; (b) mean diameter $=5.9 \mu \mathrm{m}$.

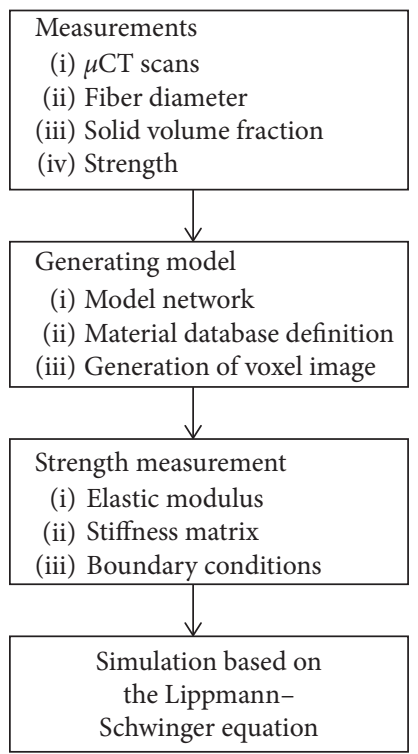

FIGURE 4: Overall scheme of the simulation used herein.

constant strain; and $\Gamma^{0}$ is to the Green operator, which is defined as $\Gamma^{0} \times \tau(x)=\int_{\Omega} \Gamma^{0}(x-y): \tau(y) \mathrm{d} y$; and $\tau(x)$ is the residual stress, and $\tau(x)=\left(C(x)-C^{0}\right): \varepsilon(x), x \in \Omega$, $C^{0}$ describes the initial stiffness, and $C(x)$ is the local stiffness.

\section{Results and Discussion}

3.1. Model Verification and Analysis. Figure 5(a) shows a cross section of the original model wherein the circular section is a slag ball and the point or line section is the fiber. Figure 5(b) is the original model with a dimension of $2 \mathrm{~mm} \times 2 \mathrm{~mm} \times 2 \mathrm{~mm}$. Figure 5(c) shows the simplified ideal model. To more clearly show the simplified fiber, the model size shown in Figure 5 (c) is $0.3 \mathrm{~mm} \times 0.3 \mathrm{~mm} \times 0.3 \mathrm{~mm}$. From Figure 5(b), it is clear that the fibers in RWB were evenly distributed and overlapped or forked. The ideal model in Figure 5(c) ignored the influence of the slag ball and equated it into the fiber. It was assumed that the fibers were randomly distributed (part 3 in Supplementary Material) and overlapped.

3.2. Compressive Strength of RWB. Figure 6 shows the measured compressive strength values of the RWBs and numerical simulation results for different SVFs. The relative error between the numerical and measured values was large for the fibers with mean diameters of 5.9 and $12 \mu \mathrm{m}$. As the SVF changed in the $10.5 \mu \mathrm{m}$ fiber diameter system, the measured value trend was consistent with simulation. Therefore, rationality of the calculation based on the L-S equation was verified. However, for the numerical simulation, assumptions regarding the slag ball and resin binder in RWB were made, and the influence of fiber curling was ignored, resulting in lower compression strength in the numerical simulation.

The mechanical properties of RWB are mainly affected by geometric parameters including fiber orientation [29], length [30], SVF, diameter [31], and contact degree among the fibers. Based on the change of geometric parameters of RWB, the influences of SVF, fiber diameter, and contact degree on mechanical properties were studied.

3.3. Constructing a Theoretical Model. The diameter distribution probability of two RWBs is shown in Figure 7.

Figures $7(a)-7(d)$ show that the different diameter distributions of RWBs with an SVF value of $4.72 \%$. Figures 7 (a) and $7(\mathrm{c})$ show the basic performance of RWB I, and Figures 7(b) and 7(d) represent RWB II. Figures 7(c) and 7 (d) shows that the mean diameter of RWB I was $10.5 \mu \mathrm{m}$ 


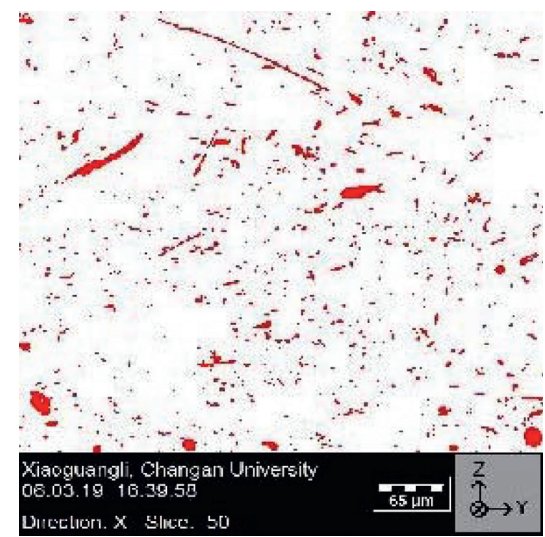

(a)

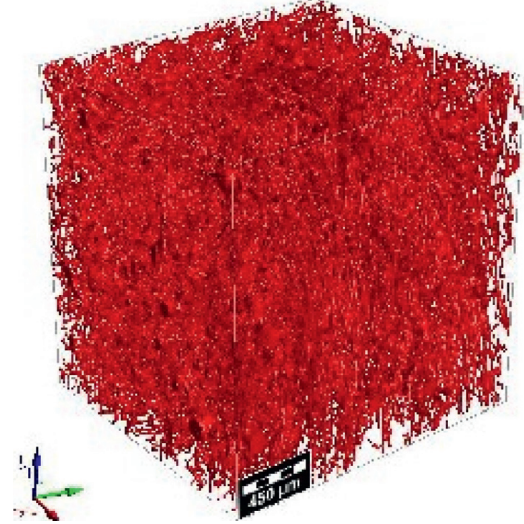

(b)

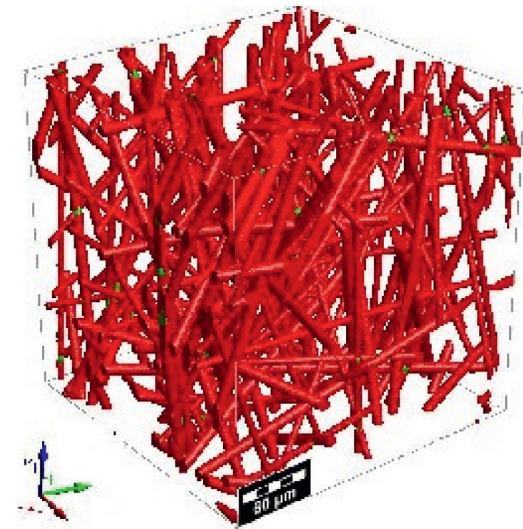

(c)

FIGURE 5: Model of the RWB: (a) cross section; (b) original model; (c) ideal model.

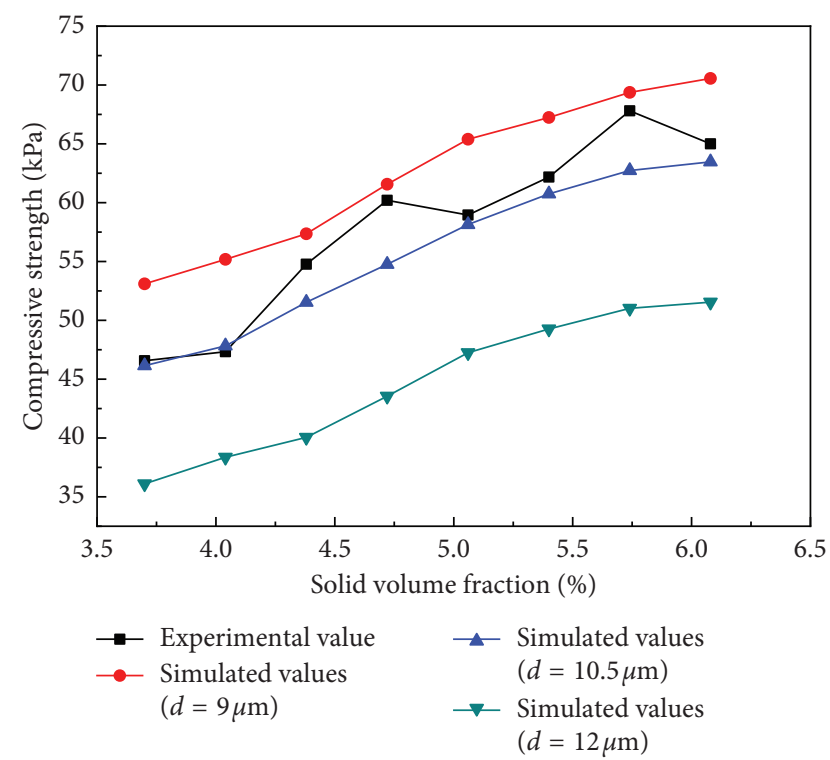

FIGURE 6: Relationship between the measured values of compressive strength and numerical calculation results of different SVF RWBs.

and that of RWB II was $5.9 \mu \mathrm{m}$. Figures 7(a) and 7(b) reflect the distribution characteristics of the fibers with different diameters. With increasing fiber diameter, the number of fibers continuously reduced in the RWB. The pore size between fibers increased, and the contact degree among the fibers was reduced weakening the bonds between fibers, which can affect the mechanical properties of RWB. Figures $7(a)-7(d)$ show that fiber diameter decreased with increasing speed of roll rotating during the fiberization process, resulting in higher SVF of the RWB and smaller pore size among fibers.

3.4. Analysis of the Influence of Fiber Diameter. It should be ensured that the model can retain the main information of real RWB at $4.72 \%$ of SVF. Figure 8 (a) shows that the RWB strengths decreased with increasing fiber diameter. When the fiber diameter ranged from 5 to $7 \mu \mathrm{m}$, the mechanical properties of RWB decreased remarkably. Compression, tensile, and shear strengths decreased by $45.4 \%, 67.6 \%$, and $81.77 \%$, respectively, with increasing fiber diameter from 3 to $10.5 \mu \mathrm{m}$. It is clear that the diameter change significantly influenced the shear strength of RWB.

Figure 9 shows that the number of fibers along with the pore size among fibers increased with increasing fiber diameter. Simultaneously, the contact surface among fibers was reduced weakening the bonds between fibers (Figure 8(b)), which is the underlying mechanism of the RWB strength decrease. In addition, the shear strength of RWB was closely related to friction area among the fibers. As the fiber diameter increased, the contact degree among fibers decreased, resulting in a lower coefficient of friction coefficient among the fibers. When the RWB was subjected to shearing, structural damage was invalidated and the strength was gradually reduced to complete failure, which was mainly due to frictional slip due to the weakened bonds between fibers [32-35]. Therefore, the lower shear strength was observed with increased fiber diameters.

These experiments also showed a negative correlation between the fiber diameter and RWB strength, as shown in Table 3. When the fiber diameter decreased by $4.6 \mu \mathrm{m}$, the compressive strength of RWB increased by $15.64 \mathrm{kPa}$ because the pore size decreased with smaller fiber diameters. In addition, the overlap among the fibers increased, thereby increasing the bond strength among fibers. Therefore, the feasibility of the simulation based on the L-S equation was verified.

3.5. Analysis of the SVF Influence. The diameter distribution in the simulation was set to a Gaussian distribution similar to the real $\mathrm{RWB}$, with a mean diameter of $10.5 \mu \mathrm{m}$. Figure 10(a) shows that the RWB strengths increased with increasing SVF [36]. The mechanical properties of RWB were greatly improved from $4.04 \%$ to $4.72 \%$ SVF. The compression, tensile, and shear strengths increased by $37.5 \%, 156.4 \%$, and $218.6 \%$, respectively, with increasing the SVF from $3.70 \%$ to $6.08 \%$. It is clear that the variation of SVF significantly influenced the shear strength of RWB. 


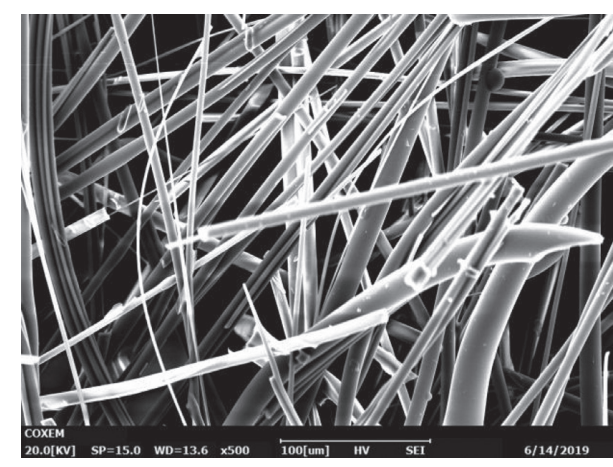

(a)

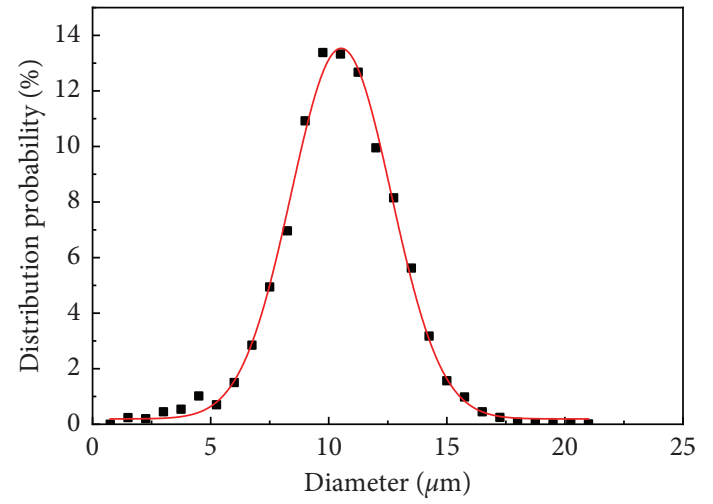

- Diameter distribution probability - Fitting curve

(c)

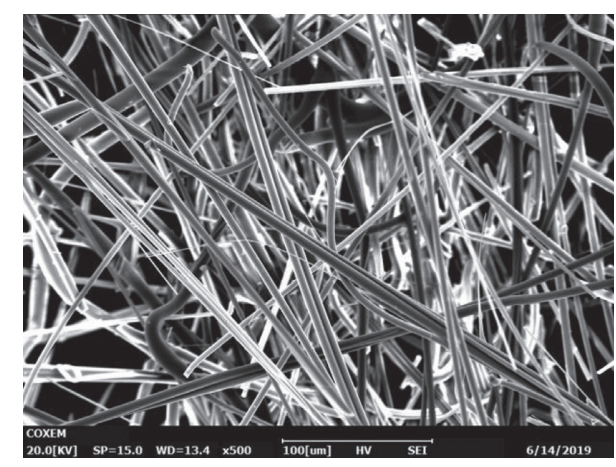

(b)

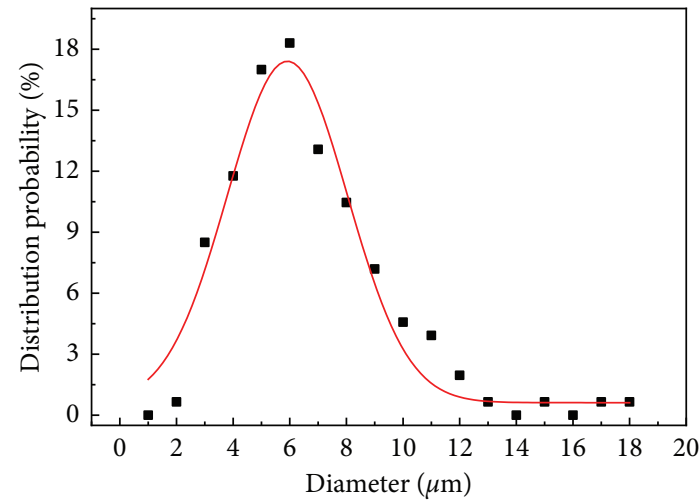

(d)

FIgURE 7: Diameter distribution probability of two RWBs.

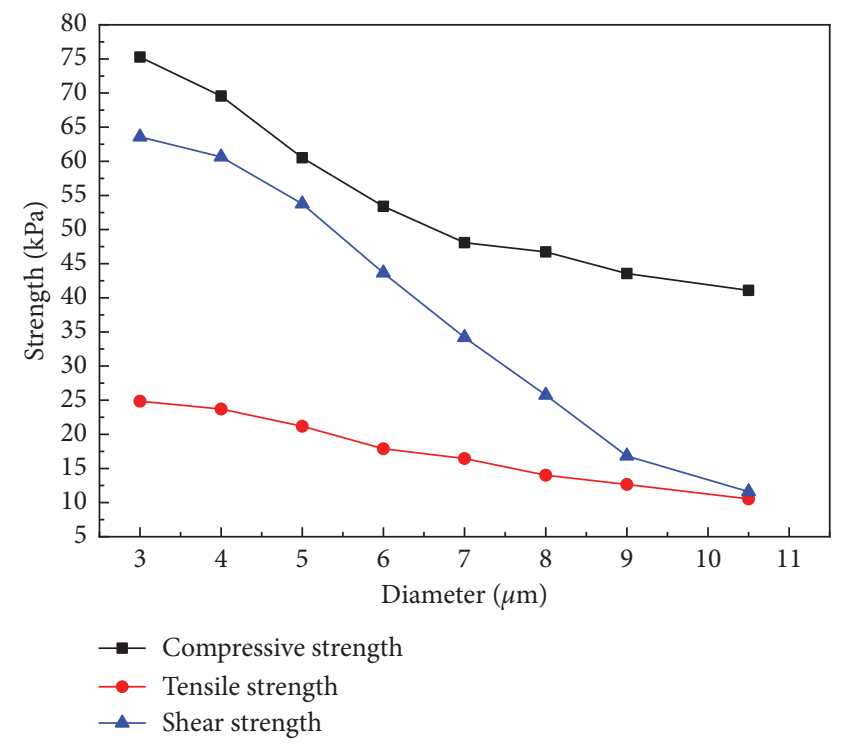

(a)

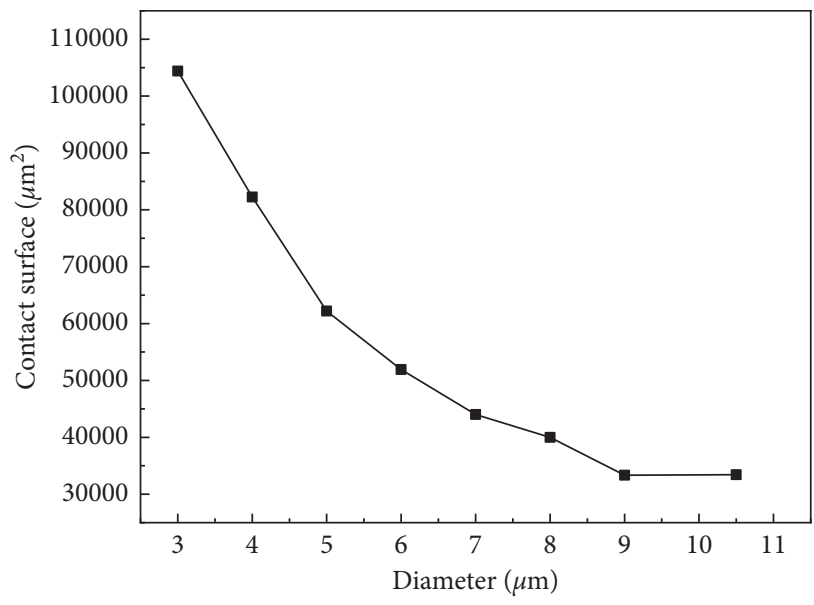

(b)

FIGURE 8: (a) Mechanical properties of RWB with different fiber diameters (SVF $=4.72 \%$ ); (b) relationship between the fiber contact area and diameter $(\mathrm{SVF}=4.72 \%)$.

Figure 11 shows that the number of fibers increased and the pore size decreased with increasing SVF. Simultaneously, the contact surface increased (Figure 10(b)), indicating increased RWB strengths due to the enhanced bonds among fibers. Similarly, the RWB shear strength was closely related to the friction surface. RWB strength was primarily 


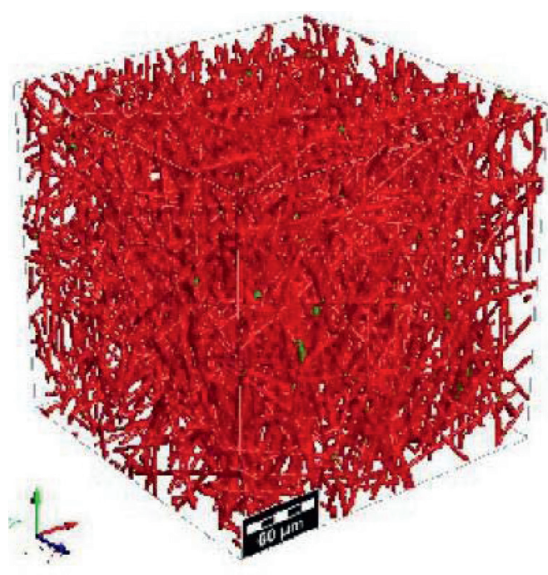

(a)

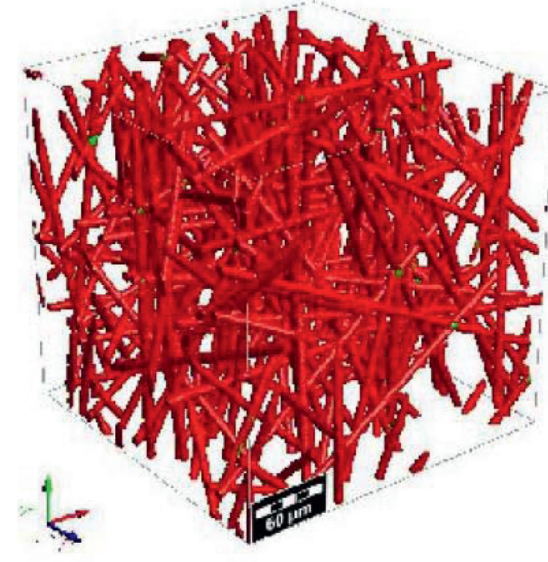

(b)

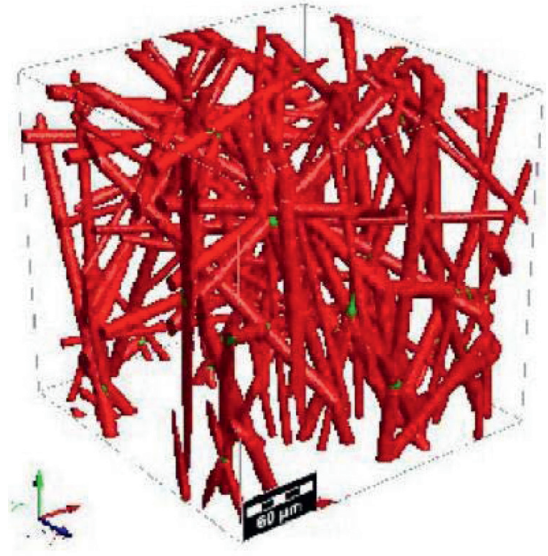

(c)

FIGURE 9: RWB model with different fiber diameter distributions (SVF $=4.72 \%$ ): (a) fiber diameter $=3 \mu \mathrm{m}$; (b) fiber diameter $=6 \mu \mathrm{m}$; (c) fiber diameter $=10.5 \mu \mathrm{m}$.

TABLE 3: Compressive strength of RWBs with different diameter distributions.

\begin{tabular}{lcc}
\hline & Mean diameter $(\mu \mathrm{m})$ & Compressive strength $(\mathrm{kPa})$ \\
\hline RWB I & 10.5 & 52.73 \\
RWB II & 5.9 & 68.37 \\
\hline
\end{tabular}

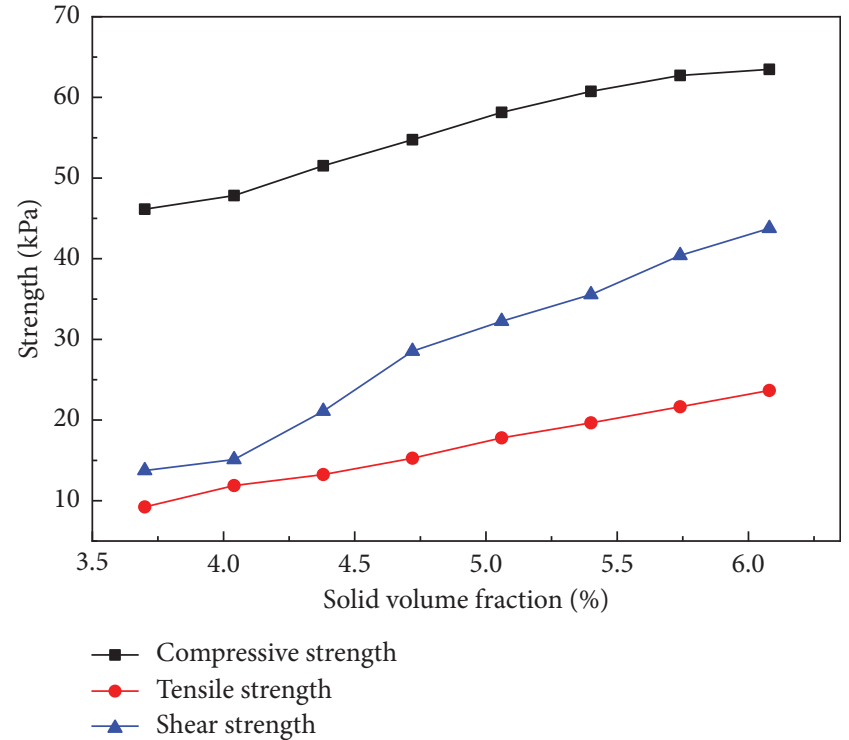

(a)

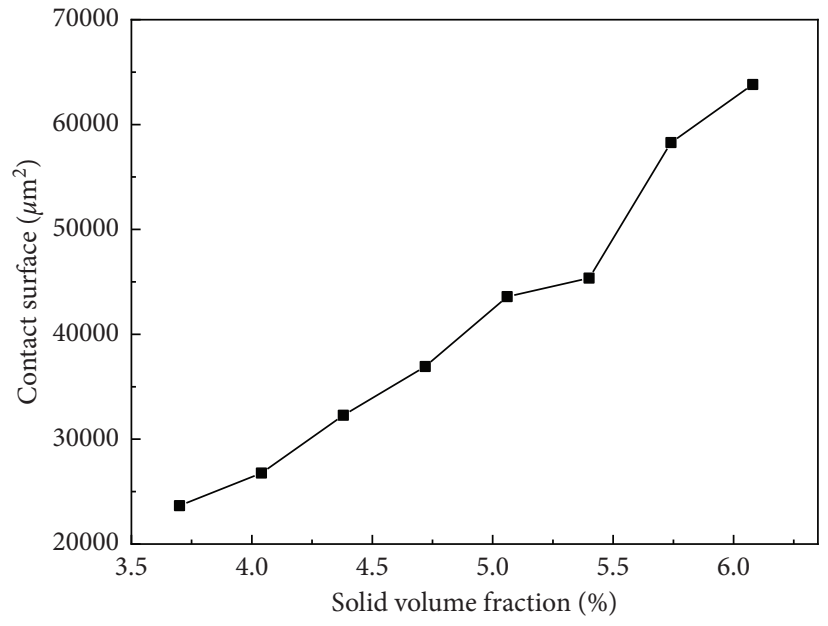

(b)

FIGURE 10: (a) Mechanical properties of RWB with different SVFs (mean diameter $=10.5 \mu \mathrm{m}$ ); (b) relationship between the fiber contact area and SVF (mean diameter $=10.5 \mu \mathrm{m})$.

controlled by its density and bond strength among fibers. Higher SVF values resulted in increased bond strength among the fibers [37]. For the sheared RWB, the frictional sliding among the fibers is smaller. The shear strength of RWB increased relatively faster than the compression and tensile strengths. The friction area in Figure 11(c) is larger than that in Figure 11(a), and the RWB exhibited a maximal shear strength value at $6.08 \%$ SVF.
Figure 12 shows the relationship between the experimentally measured RWB strengths and SVF. The compressive, tensile, and shear strengths of RWB were positively correlated with SVF. When the SVF of RWB was 3.70$6.08 \%$, the compressive strength range was $46.57-67.80 \mathrm{kPa}$; tensile strength range was $9.68-21.06 \mathrm{kPa}$; and shear strength range was $13.6-34.5 \mathrm{kPa}$. The mechanical indices increased with increasing SVF of the RWB. 


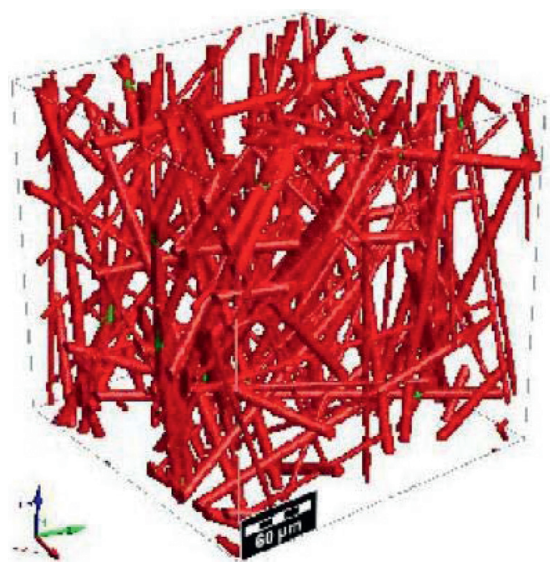

(a)

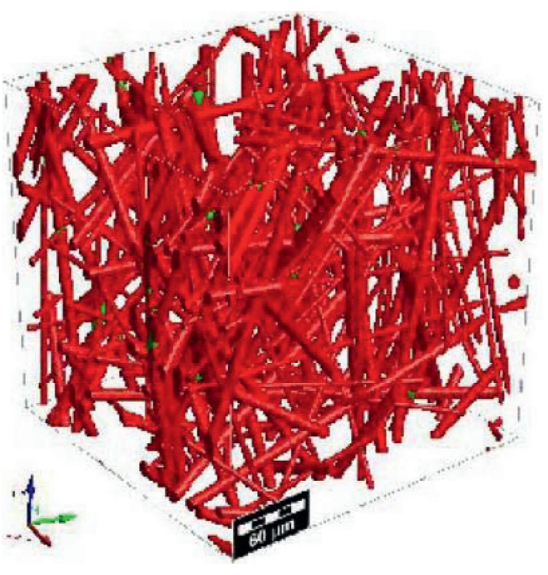

(b)

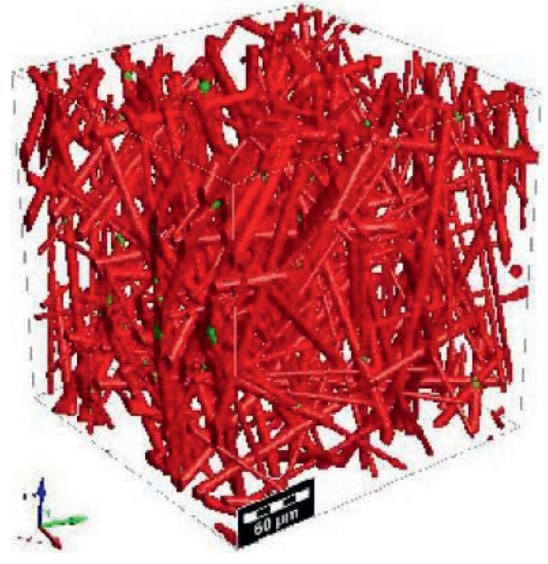

(c)

Figure 11: RWB model with different SVF distributions (mean diameter $=10.5 \mu \mathrm{m}$ ): (a) SVF=3.70\%; (b) SVF $=4.72 \%$; (c) SVF $=6.08 \%$.

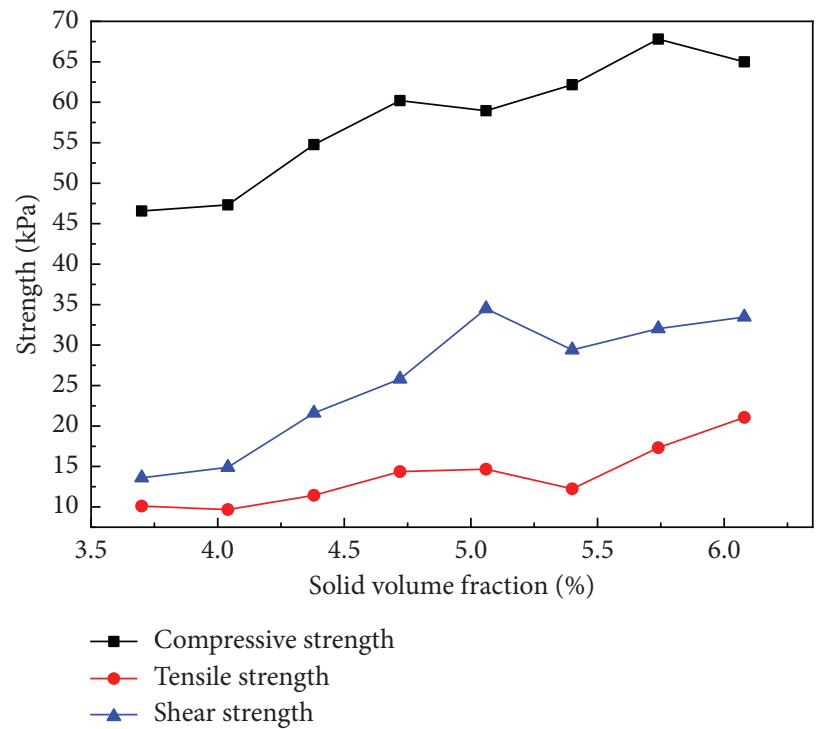

Figure 12: Testing data of the mechanical properties of RWB (mean diameter $=10.5 \mu \mathrm{m}$ ).

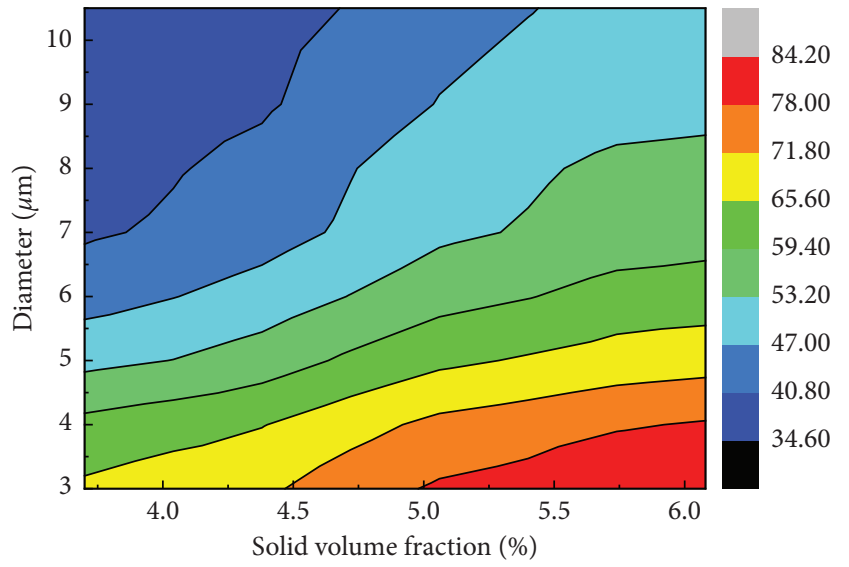

(a)

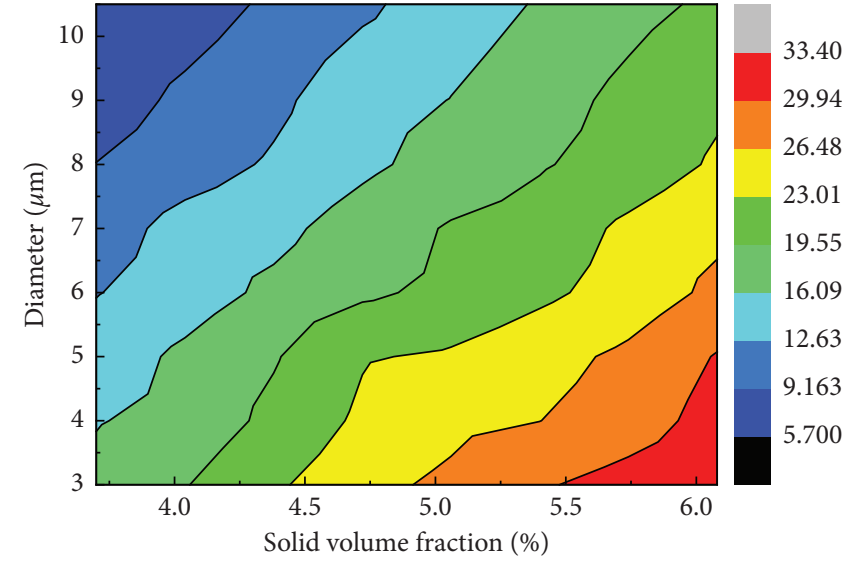

(b)

Figure 13: Continued. 


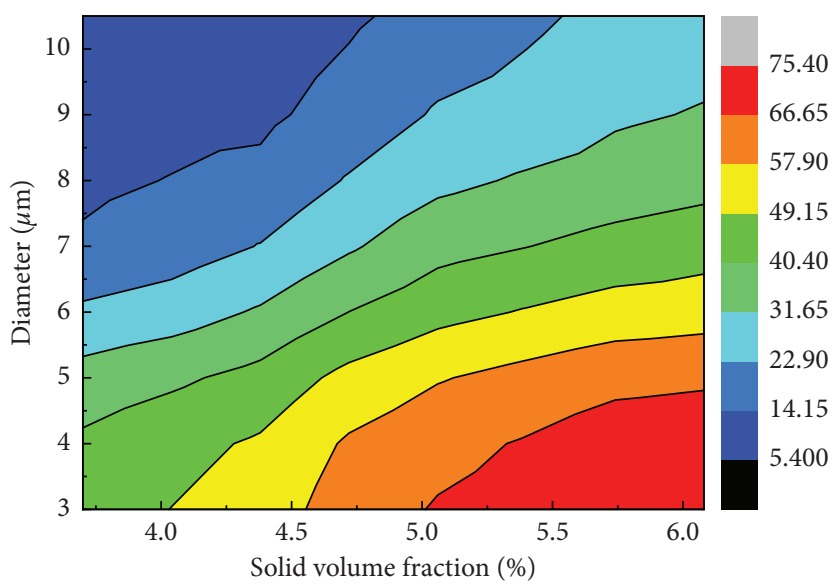

(c)

FIGURE 13: Strength under the coupling of different SVFs and fiber diameters: (a) compressive strength; (b) tensile strength; (c) shear strength.

3.6. Influence of Diameter and SVF. Figures 13(a)-13(c) show that the fiber diameter was negatively correlated with RWB strengths at a constant of SVF. When the fiber diameter was held constant, SVF was approximately positively correlated with the RWB strengths.

From Figures 13(a)-13(c), it is clear that the compression, tensile, and shear strengths of RWB increased from the lower left to upper right. As shown in Figure 13(a), when the fiber diameter was $10.5 \mu \mathrm{m}$ and SVF was $3.70 \%$, the compressive strength of RWB was at least $34.69 \mathrm{kPa}$. When the fiber diameter was $3 \mu \mathrm{m}$ and SVF was $6.08 \%$, and the maximum compressive strength was achieved at $84.14 \mathrm{kPa}$. SVF should be $\leq 4.72 \%$ when the fiber diameter of the RWB model was $10.5 \mu \mathrm{m}$, meeting the Chinese standard of $40 \mathrm{kPa}$ for compressive strength when using RWB for thermal insulation [38].

As shown in Figure 13(b), when the fiber diameter was $10.5 \mu \mathrm{m}$ and SVF was $3.70 \%$, the tensile strength of RWB was $5.73 \mathrm{kPa}$. When the fiber diameter was $3 \mu \mathrm{m}$ and SVF was $6.08 \%$, the tensile strength of RWB reached as high as $33.36 \mathrm{kPa}$. SVF should be $\leq 4.04 \%$ when the fiber diameter of the RWB model is $10.5 \mu \mathrm{m}$, exceeding the Chinese standard of $7.5 \mathrm{kPa}$.

Finally, as demonstrated in Figure 13(c), when the fiber diameter was $10.5 \mu \mathrm{m}$ and SVF was $3.70 \%$, the shear strength of RWB was at least $5.59 \mathrm{kPa}$. When the fiber diameter was $3 \mu \mathrm{m}$ and SVF was $6.08 \%$, the shear strength of RWB reached as high as $75.24 \mathrm{kPa}$. Because the Chinese standard is $20 \mathrm{kPa}$, the SVF should be $\leq 5.4 \%$ in the $10.5 \mu \mathrm{m}$ fiber diameter RWB.

In summary, fiber diameter can be controlled by the rotation speed of the four-roll high-speed centrifuge and melt viscosity during rock wool processing. According to different layer thickness and pleating degree, the SVF of RWB can be controlled to obtain RWB with different strengths, and the associated models can be used to guide actual engineering production applications.

\section{Conclusions}

The RWB strengths mainly depend on its mesostructure. According to the experimental and simulation data, the associated mechanical properties were estimated based on the analysis by using the L-S equation using GeoDict software. The main findings can be summarized as follows:

(1) A 3-D CT-scanned model was established and simplified and was based on the LippmannSchwinger equation to study the influence of different fiber diameters and SVF values on mechanical indices. Numerical simulations showed that differences between the compression strength and corresponding experimentally measured values were $<5 \%$. This verifies the accuracy of predicting the mechanical properties of RWB using this method.

(2) The SEM observations and distribution of fiber diameters indicated that as the fiber diameter in the RWB increases, the number of fibers decreases and the pore size increases.

(3) When the fiber diameter was increased from 3 to $10.5 \mu \mathrm{m}$, the mechanical properties (compression, tensile, and shear strengths) of the RWB decreased by $45.4 \%, 67.6 \%$, and $81.77 \%$, respectively. Therefore, consistent with our speculation, the fiber diameter exhibited the greatest influence on shear strength.

(4) When SVF ranged from $3.70 \%$ to $6.08 \%$, the mechanical properties (compression, tensile, and shear strengths) of RWB increased by $37.5 \%, 156.4 \%$, and $218.6 \%$, respectively. Therefore, SVF showed the greatest influence on the shear strength, which is consistent with the hypothesis.

(5) The mechanical features of RWB with different fiber diameters and SVF values were studied. According to the Chinese standards for compression, tensile, and shear strengths, SVF should be $\leq 4.72 \%, \leq 4.04 \%$, and $\leq 5.4 \%$, respectively. With a mean fiber diameter of $10.5 \mu \mathrm{m}$, all mechanical performance requirements for insulation materials can be satisfied. 
In field applications, fiber diameter can be controlled by the rotating speed of the centrifuge and melt viscosity, whereas the SVF can be regulated by layer thickness. Therefore, it is possible to design RWBs with different mechanical strengths by adjusting the SVF and fiber diameter to meet different requirements.

\section{Data Availability}

The data required to reproduce these findings are available from the corresponding author upon request.

\section{Conflicts of Interest}

The authors declare that there are no conflicts of interest.

\section{Acknowledgments}

This work was financially supported by the Fundamental Research Funds for the Central Universities (No. 310828152016), and Shaanxi Provincial Science and Technology Co-Ordination and Innovation Project (No. 2013KTCG02-02).

\section{Supplementary Materials}

This includes five parts: the first part is the graphic abstract and its description, followed by the distribution of fibers at different directions in the model. The third one is the basis of fiber orientation setting, and the fourth is the distribution of contact surface among fibers. The last one is the fitting relationship between fiber diameter, SVF, and mechanical properties of RWB. (Supplementary Materials)

\section{References}

[1] S. Staub, H. Andrä, and M. Kabel, "Fast FFT based solver for rate-dependent deformations of composites and nonwovens," International Journal of Solids and Structures, vol. 154, pp. 33-42, 2016.

[2] S. J. Eichhorn and R. J. Young, "Deformation micromechanics of natural cellulose fibre networks and composites," Composites Science and Technology, vol. 63, no. 9, pp. 1225-1230, 2003.

[3] J. X. Liu, Z. T. Chen, H. Wang, and K. C. Li, "Elasto-plastic analysis of influences of bond deformability on the mechanical behavior of fiber networks," Theoretical and Applied Fracture Mechanics, vol. 55, no. 2, pp. 131-139, 2011.

[4] D. V. Wilbrink, L. A. A. Beex, and R. H. J. Peerlings, "A discrete network model for bond failure and frictional sliding in fibrous materials," International Journal of Solids and Structures, vol. 50, no. 9, pp. 1354-1363, 2013.

[5] S. R. Guo, "The application and developmental trend of exterior wall external insulation," Applied Mechanics and Materials, vol. 174-177, pp. 1367-1371, 2012.

[6] L. I. Zhu, B. Yuan, R. Wang, and H. J. Zhou, "Development of the wall with insulation plates and countermeasures to its shortcomings in China," Building Energy Efficiency, vol. 39, no. 8, pp. 35-37, 2011.

[7] I. Gnip, S. Vejjelis, V. Keršulis, and S. Vaitkus, "Strength and deformability of mineral wool slabs under short-term compressive, tensile and shear loads," Construction and Building Materials, vol. 24, no. 11, pp. 2124-2134, 2010.
[8] A. Buska and R. Mačiulaitis, "The compressive strength properties of mineral wool slabs: influence of structure anisotropy and methodical factors," Journal of Civil Engineering and Management, vol. 13, no. 2, pp. 97-106, 2007.

[9] F. Hild, E. Maire, S. Roux, and J.-F. Witz, "Three-dimensional analysis of a compression test on stone wool," Acta Materialia, vol. 57, no. 11, pp. 3310-3320, 2009.

[10] G. B. Olson, "Computational design of hierarchically structured materials," Science, vol. 277, no. 5330, pp. 1237-1242, 1997.

[11] H. Xu, D. A. Dikin, C. Burkhart, and W. Chen, "Descriptorbased methodology for statistical characterization and 3D reconstruction of microstructural materials," Computational Materials Science, vol. 85, no. 4, pp. 206-216, 2014.

[12] National Research Council, Integrated Computational Materials Engineering: A Transformational Discipline for Improved Competitiveness and National Security, National Academies Press, Washington, DC, USA, 2008.

[13] K. Schladitz, S. Peters, D. Reinel-Bitzer, A. Wiegmann, and J. Ohser, "Design of acoustic trim based on geometric modeling and flow simulation for non-woven," Computational Materials Science, vol. 38, no. 1, pp. 56-66, 2007.

[14] J. Feng, Q. Teng, X. He, L. Qing, and Y. Li, "Reconstruction of three-dimensional heterogeneous media from a single twodimensional section via co-occurrence correlation function," Computational Materials Science, vol. 144, pp. 181-192, 2018.

[15] U. Bonse, R. Nusshardt, F. Busch, J. H. Kinney, R. A. Saroyan, and M. C. Nichols, "X-ray tomographic microscopy," in $X$ Ray Microscopy III, Springer, Berlin, Germany, 1992.

[16] P.-C. Gervais, S. Bourrous, F. Dany, L. Bouilloux, and L. Ricciardi, "Simulations of filter media performances from microtomography-based computational domain. Experimental and analytical comparison," Computers \& Fluids, vol. 116, pp. 118-128, 2015.

[17] N. Chawla and K. K. Chawla, "Microstructure-based modeling of the deformation behavior of particle reinforced metal matrix composites," Journal of Materials Science, vol. 41, no. 3, pp. 913-925, 2006.

[18] N. Chawla, V. V. Ganesh, and B. Wunsch, "Three-dimensional (3D) microstructure visualization and finite element modeling of the mechanical behavior of $\mathrm{SiC}$ particle reinforced aluminum composites," Scripta Materialia, vol. 51, no. 2, pp. 161-165, 2004.

[19] A. Wanko, J. Laurent, P. Bois et al., “Assessment of rock wool as support material for on-site sanitation: hydrodynamic and mechanical characterization," Environmental Technology, vol. 37, no. 3, pp. 369-380, 2016.

[20] B. Pourdeyhimi, B. Mazé, and H. V. Tafreshi, "Simulation and analysis of unbonded nonwoven fibrous structures," Journal of Engineered Fibers \& Fabrics, vol. 1, no. 2, pp. 47-65, 2006.

[21] R. Arambakam, H. Vahedi Tafreshi, and B. Pourdeyhimi, "Analytical monte carlo ray tracing simulation of radiative heat transfer through bimodal fibrous insulations with translucent fibers," International Journal of Heat \& Mass Transfer, vol. 55, no. 23-24, pp. 7234-7246, 2012.

[22] X. Li, J. Liu, J. Qin, Y. Liu, Y. Qu, and R. Zhang, "Dissolution behavior of a novel composite fiber made from blast furnace slag," Construction and Building Materials, vol. 206, pp. 442-448, 2019.

[23] B. Širok, B. Bizjan, A. Orbanie, and T. Bajcar, "Mineral wool melt fiberization on a spinner wheel," Chemical Engineering Research \& Design, vol. 92, no. 1, pp. 80-90, 2014.

[24] Y. Zhang, Y. Vulfson, Q. Zheng, J. Luo, S. H. Kim, and Y. Yue, "Impact of fiberizing method on physical properties of glass 
wool fibers," Journal of Non-Crystalline Solids, vol. 476, pp. 122-127, 2017.

[25] F. Trdič, B. Širok, P. R. Bullen, and D. R. Philpott, "Monitoring mineral wool production using real-time machine vision," Real-Time Imaging, vol. 5, no. 2, pp. 125-140, 1999.

[26] T. Westerlund and T. Hoikka, "On the modeling of mineral fiber formation," Computers \& Chemical Engineering, vol. 13, no. 10, pp. 1153-1163, 1989.

[27] P. P. Castañeda and P. Suquet, "Nonlinear composites," Advances in Applied Mechanics, vol. 34, no. 34, pp. 171-302, 1997.

[28] S. Nemat-Nasser, M. Lori, and S. K. Datta, "Micromechanics: overall properties of heterogeneous materials," Journal of Applied Mechanics, vol. 63, no. 2, p. 561, 1996.

[29] S. Fotovati, H. Vahedi Tafreshi, and B. Pourdeyhimi, "Influence of fiber orientation distribution on performance of aerosol filtration media," Chemical Engineering Science, vol. 65, no. 18, pp. 5285-5293, 2010.

[30] Q. Wang, B. Maze, H. V. Tafreshi, and B. Pourdeyhimi, "Simulating through-plane permeability of fibrous materials with different fiber lengths," Modelling and Simulation in Materials Science and Engineering, vol. 15, no. 8, pp. 855-868, 2007.

[31] S. A. Hosseini and H. V. Tafreshi, "3-D simulation of particle filtration in electrospun nanofibrous filters," Powder Technology, vol. 201, no. 2, pp. 153-160, 2010.

[32] S. Heyden, Network Modelling for Evaluation of Mechanical Properties of Cellulose Fibre Fluff, Report Tvsm, Lund University, Lund, Sweden, 2000.

[33] A. Ridruejo, C. González, and J. LLorca, "Damage micromechanisms and notch sensitivity of glass-fiber non-woven felts: an experimental and numerical study," Journal of the Mechanics and Physics of Solids, vol. 58, no. 10, pp. 1628-1645, 2010.

[34] A. Ridruejo, C. González, and J. LLorca, "Micromechanisms of deformation and fracture of polypropylene nonwoven fabrics," International Journal of Solids and Structures, vol. 48, no. 1, pp. 153-162, 2011.

[35] A. Kulachenko and T. Uesaka, "Direct simulations of fiber network deformation and failure," Mechanics of Materials, vol. 51, pp. 1-14, 2012.

[36] M. Henriksson, L. A. Berglund, P. Isaksson, T. Lindström, and T. Nishino, "Cellulose nanopaper structures of high toughness," Biomacromolecules, vol. 9, no. 6, pp. 1579-1585, 2008.

[37] S. Goutianos, R. Mao, and T. Peijs, "Effect of inter-fibre bonding on the fracture of fibrous networks with strong interactions," International Journal of Solids and Structures, vol. 136-137, pp. 271-278, 2018.

[38] J. L. Guo, Q. Du, and L. Lu, Application of Rock-Wool in Outer-Wall External Thermal Insulation System, Advanced Materials Research, Trans Tech Publications, Stafa-Zurich, Switzerland, 2013. 